\title{
Short-term and Medium-term Outcomes of Low Midline and Low Transverse Incisions in Laparoscopic Rectal Cancer Surgery
}

\author{
Do Hoe Ku, Hyeon Seung Kim, Jin Yong Shin \\ Department of Surgery, Inje University Haeundae Paik Hospital, Inje University College of Medicine, Busan, Korea
}

\begin{abstract}
Purpose: Limited data exist on the use of low midline and transverse incisions for specimen extraction or stoma sites in laparoscopic rectal cancer surgery (LRCS). We compared the short-term and medium-term outcomes of these incisions and assessed whether wound complications in specimen extraction sites (SES) are increased by specimen extraction through the stoma site (SESS) in LRCS.

Methods: From March 2010 to December 2017, 189 patients who underwent LRCS and specimen extraction through low abdominal incisions were divided into 2 groups: midline $(n=102)$ and transverse $(n=87)$, and perioperative outcomes were compared.

Results: The midline group showed a higher frequency of temporary stoma formation $(\mathrm{P}=0.001)$ and splenic flexure mobilization $(\mathrm{P}<0.001)$ than the transverse group. The overall incisional hernia and wound infection rates in the SES were $21.6 \%$ and $25.5 \%$, respectively, in the midline group and $26.4 \%$ and $17.2 \%$, respectively, in the transverse group $(\mathrm{P}=0.494$ and $\mathrm{P}=0.232$, respectively). In patients who underwent SESS, the incisional hernia and wound infection rates of SES after stoma closure were $39.1 \%$ and $43.5 \%$, respectively, in the midline group, and $35.5 \%$ and $22.6 \%$, respectively, in the transverse group ( $\mathrm{P}=0.840$ and $\mathrm{P}=0.035$, respectively).

Conclusion: In terms of incisional hernia and wound infection at the SES, a low midline incision may be used as a low transverse incision in patients without temporary stoma in LRCS. However, considering the high wound complication rates after stoma closure in patients with SESS in this study, SESS should be performed with caution in LRCS.
\end{abstract}

Keywords: Rectal neoplasms; Outcomes; Incisions; Specimen extraction

\section{INTRODUCTION}

A mini-laparotomy for specimen extraction following laparoscopic colorectal surgery is usually performed through a transverse or midline incision of the abdomen $[1,2]$. However, poten-

Received: Sep 10, 2019 - Revised: Oct 16, 2019 • Accepted: Oct 22, 2019 Correspondence to: Jin Yong Shin, M.D.

Department of Surgery, Inje University Haeundae Paik Hospital, Inje University College of Medicine, 875 Haeun-daero, Haeundae-gu, Busan 48108, Korea

Tel: +82-51-797-0656, Fax: +82-51-797-0260

E-mail: jyong@paik.ac.kr

ORCID: https://orcid.org/0000-0002-1760-8376

(C) 2020 The Korean Society of Coloproctology

This is an open-access article distributed under the terms of the Creative Commons Attribution NonCommercial License (https://creativecommons.org/licenses/by-nc/4.0) which permits unrestricted non-

commercial use, distribution, and reproduction in any medium, provided the original work is properly cited. tial complications, such as incisional hernia, wound infection, and tumor seeding to the wound, may occur [2-4]. Although some studies have reported that low transverse and umbilical incisions as specimen extraction sites (SES) have a similar incisional hernia and wound infection rates after laparoscopic colorectal surgery [5-7], a low transverse incision is recommended because the incisional hernia rate is lower than in midline incision [1-3, 8-10].

Laparoscopic rectal cancer surgery (LRCS) has been advantageous in postoperative recovery, and with oncologic results similar to those of open surgery in large randomized studies [11, 12]. Thus, the application of this procedure is increasing [13]. Specimen extraction following LRCS is possible through the anus [1416], but in most cases, it is achieved through the low transverse abdomen, midline abdomen, or potential stoma site [1, 5, 17-19]. Some studies [17-19] have also attempted specimen extraction through a stoma site (SESS) to minimize additional mini-laparot- 
omy for a temporary diverting stoma in LRCS. Recent studies [20, 21] have reported the feasibility of SESS utilizing umbilicus in LRCS. Based on these studies [17-21], a low midline incision with benefits in respiratory function, pain intensity [22], and minimizing additional mini-laparotomy for a temporary diverting stoma may also be considered for the SES during LRCS. Previous reports [17-19] about specimen extraction or stoma sites in LRCS focus only on the short-term results of a low transverse incision, and thus are lacking data on low midline incisions or medium-term results, such as the incidence of incisional hernia and wound infection, at the SES after stoma closure in patients who undergo SESS.

Thus, the aim of this study was to compare the results of low midline and low transverse incisions and to evaluate the usefulness of a low midline incision in LRCS. Furthermore, we assessed whether the wound complications at SES are increased by SESS in LRCS.

\section{METHODS}

The Institutional Review Board of Inje University Haeundae Paik Hospital approved the study protocol (No. HPIRB 2019-07-025002 ) and written informed consent was obtained from all patients before surgery.

A total of 330 consecutive rectal cancer patients who underwent laparoscopic surgery between March 2010 and December 2017 were identified from the colorectal cancer databases recorded in a prospective manner at the authors' institutions. Of the 330 patients, the following 141 patients were excluded: (1) specimen extracted through upper midline, transumbilical, and Pfannenstiel incision ( $\mathrm{n}=77)$; (2) patients who did not undergo mini-laparotomy incision due to totally laparoscopic rectal resection $(n=18)$;

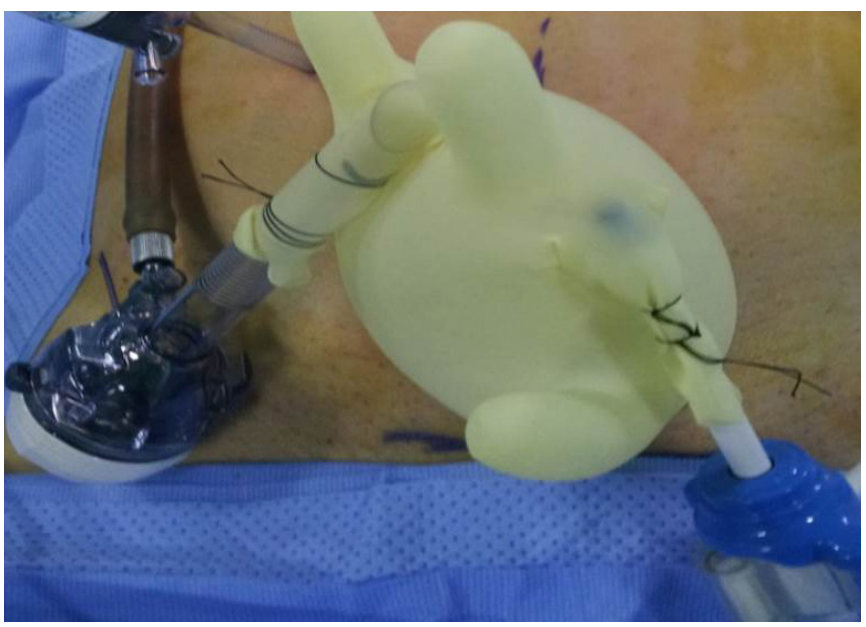

Fig. 1. Home-made "glove port". One 5-mm and one 12-mm trocar were placed in 2 surgical glove fingers and attached to the wound protector.
(3) patients without temporary stoma closure $(n=18)$; (4) patients who were not followed up immediately after surgery $(n=14) ;(5)$ patients who underwent abdominoperineal resection or Hartmann type resection $(n=10)$; and (6) conversion to open surgery $(n=4)$. A total of 189 patients excluding the above-mentioned were enrolled in this study. A low midline incision was preferentially performed in patients with a relatively short distance from the symphysis pubis to the umbilicus or a previous low midline incision. The patients were divided into 102 who underwent LRCS through a low midline incision (midline group) and 87 who underwent LRCS through a right or left low transverse incision (transverse group) as the SES. The clinical characteristics of the patients and perioperative outcomes were analyzed retrospectively. Surgery was performed by a single colorectal surgeon and the first mini-laparotomy was performed according to tumor size in a similar manner to single port laparoscopic surgery. The wound was applied with an Alexis wound protector (Applied Medical, Rancho Santa Margarita, CA, USA). One 5-mm and one 12-mm trocar were placed in 2 surgical glove fingers ('glove port') and attached to the wound protector (Fig. 1). Following pneumoperitoneum, a 5-mm laparoscopic camera port was inserted in the umbilicus and an additional port was placed in the right or left abdomen according to the abdominal incision (Fig. 2). The brief operative procedure was as follows: the inferior mesenteric artery was mostly high ligated, and the left colon was separated from the left retroperitoneum. The rectum was mobilized down to the pelvic floor muscle level or below. After washing the rectum below the tumor with a povidone solution, the rectum was cut through a $12-\mathrm{mm}$ port located in the glove port. Following specimen extraction, the anastomosis of the intestinal tract was performed using a circular stapler and a reinforcement suture at the anastomotic site was performed intracorporeally. When anastomosis using a cir-

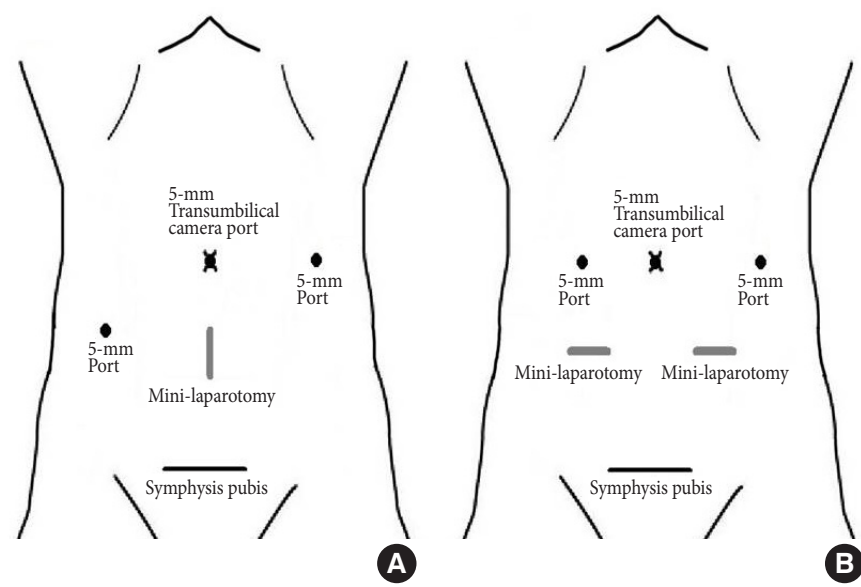

Fig. 2. (A) Port placement for patients who underwent laparoscopic rectal cancer surgery (LRCS) using a low midline incision. (B) Port placement for patients who underwent LRCS using a low transverse incision. 
cular stapler was difficult, hand-sewn coloanal anastomosis was performed. Temporary diverting stoma was created at the discretion of the surgeon in cases of very low anastomosis (anastomosis within 2 to $3 \mathrm{~cm}$ of the anal verge), difficult pelvic dissection, positive air leak test, poor bowel preparation, or the poor general condition of patients. A temporary stoma was created via a low midline incision with SESS or a separate right lower transverse incision with no SESS in the midline group, and a right (left) lower transverse incision with SESS or a separate right (left) upper transverse incisions in the transverse group. When a difference in size exists between the stoma and the mini-laparotomy incision, the SES was adjusted to the size of the stoma by peristomal suture.

On the day before surgery, oral antibiotics (metronidazole and tetracycline), and sodium picosulfate or bisacodyl were used for bowel preparation. Intravenous patient-controlled anesthesia with fentanyl was used for postoperative pain management. Most abdominal incisions were closed by the operating surgeon with a polyglactin using an interrupted method. Abdominal closures after stoma closure were performed using a single layer interrupted closure technique of the fascia and peritoneum with polyglactin in both groups. Until 2011, conventional primary linear sutures were used for skin closure; however, since 2012, a single-layer subcutaneous suture using a semi-closed method was performed with gauze dressing in both groups. In most patients who underwent stoma closure, a wound drainage tube was not inserted.

The amount of blood loss during surgery was calculated by subtracting the volume of instilled fluids from the volume of aspirated fluids. Postoperative complications occurring within 30 days after surgery, except for incisional hernia, were included and graded using the Clavien-Dindo classification. An incisional hernia was defined as an abdominal wall gap in the SES, trocar site, and additional stoma site by using abdominal computed tomography. It was evaluated after stoma closure in patients with a temporary stoma. Diagnosis of wound infection was considered positive when any of the following events occurred: purulent discharge; positive wound culture; clinical symptoms or signs, such as pain, erythema, and edema; and wound opening by the surgeon for wound debridement or wound drainage. In the event of wound infection, the wound was managed by wound opening and wound debridement, if necessary, at the bedside during hospitalization or the outpatient clinic, and allowed to heal by a second intention. Wound infection was graded according to classification by Dindo et al. [23]. Grade I infection was defined as a wound infection treated by a wound opening. Grade II infection was defined as a wound infection requiring antibiotic treatment because of additional phlegmonous infection.

\section{Statistics}

Statistical analysis was performed using SPSS ver. 17.0 (SPSS Inc., Chicago, IL, USA). Categorical variables were analyzed using a chi-square or Fisher exact test, and continuous variables were tested by independent $\mathrm{t}$-test. A P-value less than 0.05 was consid- ered statistically significant.

\section{RESULTS}

Comparison of clinical variables in both groups of patients The mean values of the body mass index were 23.7 and $24.2(\mathrm{P}=$ $0.344)$, with a mean age of 64.3 and 64.9 years $(\mathrm{P}=0.733)$ in the midline and transverse groups, respectively. The incidence of tumors located below the anterior peritoneal reflection was $52.0 \%$, and the abdominal operation history was $24.5 \%$ in the midline group. No statistically significant difference was found in tumor size, preoperative radiotherapy frequency, and stage distribution between the 2 groups (Table 1 ).

\section{Short-term outcomes following surgery}

The mean length of the mini-laparotomy was $4.5 \mathrm{~cm}$ in the midline group, but it was not significantly different from $4.2 \mathrm{~cm}$ in the transverse group $(\mathrm{P}=0.874)$. The mean values of operation time and blood loss were 284.3 minutes and $156.0 \mathrm{~mL}$, respectively, and no statistically significant difference was found between the 2 groups. Moreover, no significant difference was noted in the incidence of circumferential resection margin involvement and adequate distal resection margin in the midline and transverse groups $(\mathrm{P}=0.548, \mathrm{P}=0.154)$. The incidence of additional abdominal incisions for the temporary stoma was $8.0 \%$ in the midline group and $3.1 \%$ in the transverse group $(\mathrm{P}=0.231)$. There was a lower rate of temporary stoma formation $(\mathrm{P}=0.001)$ and a higher rate of splenic flexure mobilization $(\mathrm{P}<0.001)$ in the midline

Table 1. Clinicopathologic features of patients classified according to the mini-laparotomy incision site

\begin{tabular}{lccc}
\hline Variable & $\begin{array}{c}\text { Midline } \\
\text { group } \\
(\mathrm{n}=102)\end{array}$ & $\begin{array}{c}\text { Transverse } \\
\text { group } \\
(\mathrm{n}=87)\end{array}$ & P-value \\
\hline Age (yr) & $64.3 \pm 11.2$ & $64.9 \pm 10.7$ & 0.733 \\
Male sex & $63(61.8)$ & $58(66.7)$ & 0.544 \\
ASA PS classification III \& IV & $17(16.7)$ & $20(23.0)$ & 0.358 \\
Body mass index (kg/m²) & $23.7 \pm 3.5$ & $24.2 \pm 3.6$ & 0.344 \\
Previous abdominal surgery & $25(24.5)$ & $21(23.9)$ & 1.000 \\
Preoperative chemoradiation & $10(9.8)$ & $5(5.7)$ & 0.420 \\
Lower rectal tumor ${ }^{\mathrm{a}}$ & $53(52.0)$ & $49(56.3)$ & 0.562 \\
Tumor size (cm) & $3.7 \pm 1.5$ & $3.6 \pm 1.5$ & 0.355 \\
Stage & & & 0.186 \\
$\quad$ 0-II & $53(52.0)$ & $54(62.1)$ & \\
III-IV & $49(48.0)$ & $33(37.9)$ & \\
\hline
\end{tabular}

Values are presented as mean \pm standard deviation or number (\%) unless otherwise denoted.

ASA, American Society of Anesthesiologists; PS, physical status.

aLower rectal tumor was defined as a tumor located below the anterior peritoneal reflection. 
group than in the transverse group (Table 2).

Postoperative recovery variables, such as first gas or stool passage, postoperative pain score, and length of hospital stay, were similar in the midline and the transverse groups (Table 3).

\section{Postoperative complications}

Among the Clavien-Dindo complications classified as mild grade I or II, postoperative voiding dysfunction was the most common in both groups (12.7\% in the midline group and $11.5 \%$ in the transverse group). Purulent or turbid discharge was released from the abdominal drainage tube in 9 patients in the midline and transverse groups. Severe complications of Clavien-Dindo grade III or higher occurred in 3 patients (2.9\%) in the midline group and in 3 patients $(3.4 \%)$ in the transverse group $(\mathrm{P}=0.827)$. The

Table 2. Comparison of operative results of patients classified according to the mini-laparotomy incision site

\begin{tabular}{lccc}
\hline Variable & $\begin{array}{c}\text { Midline } \\
\text { group } \\
(\mathrm{n}=102)\end{array}$ & $\begin{array}{c}\text { Transverse } \\
\text { group } \\
(\mathrm{n}=87)\end{array}$ & P-value \\
\hline Length of mini-laparotomy incision (cm) & $\begin{array}{c}4.5 \pm 1.0 \\
284.3 \pm 75.3\end{array}$ & $\begin{array}{c}4.2 \pm 0.7 \\
\text { Operation time (min) }\end{array}$ & 0.874 \\
Estimated blood loss (mL) & $156.0 \pm 121.6$ & $191.6 \pm 201.8$ & 0.141 \\
Proximal resection margin $\geq 10 \mathrm{~cm}$ & $100(98.0)$ & $86(98.9)$ & 1.000 \\
Distal resection margin $\geq 1 \mathrm{~cm}$ & $85(83.3)$ & $65(74.7)$ & 0.154 \\
Temporary stoma formation & $50(49.0)$ & $64(73.6)$ & 0.001 \\
Additional mini-laparotomy incision for & $4 / 50$ & $2 / 64$ & 0.231 \\
temporary stoma ${ }^{\mathrm{a}}$ & & & \\
Splenic flexure mobilization & $55(53.9)$ & $21(24.1)$ & $<0.001$ \\
Dissected nodes & $25.2 \pm 12.7$ & $22.6 \pm 11.1$ & 0.138 \\
CRM involvement(\%) & $4(3.9)$ & $4(4.6)$ & 0.548 \\
\hline
\end{tabular}

Values are presented as mean \pm standard deviation or number (\%) unless otherwise denoted.

CRM, circumferential resection margin.

${ }^{a}$ Calculated in only patients who underwent temporary stoma. ${ }^{\mathrm{b}} \mathrm{CRM}$ involvement was defined as tumor observed less than $1 \mathrm{~mm}$ from the nonperitoneal resection margin.

Table 3. Postoperative recovery outcomes of patients classified according to the mini-laparotomy incision site

\begin{tabular}{lccc}
\hline Variable & $\begin{array}{c}\text { Midline } \\
\text { group } \\
(\mathrm{n}=102)\end{array}$ & $\begin{array}{c}\text { Transverse } \\
\text { group } \\
(\mathrm{n}=87)\end{array}$ & P-value \\
\hline Time to first flatus or stool passage (day) & $2.6 \pm 2.0$ & $2.5 \pm 1.5$ & 0.786 \\
Postoperative pain score, VAS $^{\text {a }}$ & $5.6 \pm 1.1$ & $5.7 \pm 1.6$ & 0.647 \\
Postoperative length of hospital stay (day) & $15.2 \pm 6.5$ & $16.2 \pm 7.5$ & 0.264 \\
\hline
\end{tabular}

Values are presented as mean \pm standard deviation unless otherwise denoted. VAS, visual analog scale.

aMaximum VAS scores on days 1 to 3 after surgery were used to assess postoperative pain severity. incidence of overall complications within 30 days after surgery was similar in both groups $(\mathrm{P}=0.780)$ (Table 4$)$.

\section{Wound complications in SES}

The overall incisional hernia rate was $21.6 \%$ in the midline group and $26.4 \%$ in the transverse incision group $(\mathrm{P}=0.494)$, with a median follow-up period of 31 months (range, 4 to 90 months). No incisional hernia or wound infection occurred except in the SES. In patients who did not undergo temporary stoma, the incisional hernia rate of the SES was $7.1 \%$ in the midline group and $4.0 \%$ in the transverse group $(\mathrm{P}=0.507)$. The wound infection rate after stoma closure in patients with SESS was $43.5 \%$ in the midline group and $22.6 \%$ in the transverse group $(\mathrm{P}=0.035)$. Wound complications in relation to whether the specimens were extracted through the stoma site were compared. In patients whose incisions were only used for specimen extraction $(n=81)$ the incisional hernia and wound infection rates of the SES were $6.2 \%$ and $8.6 \%$, respectively, and in patients who underwent SESS $(n=108)$ the incisional hernia and wound infection rates of the SES after stoma closure were $37.0 \%$ and $31.5 \%$, respectively (both $\mathrm{P}<$ 0.001 ). However, the rate of the grade II wound infection in the

Table 4. Clavien-Dindo classification of early complications of patients classified according to the mini-laparotomy incision site

\begin{tabular}{|c|c|c|c|}
\hline Variable & $\begin{array}{l}\text { Midline } \\
\text { group } \\
(n=102)\end{array}$ & $\begin{array}{c}\text { Transverse } \\
\text { group } \\
(\mathrm{n}=87)\end{array}$ & P-value \\
\hline Grade I \& II & $36(35.3)$ & $32(36.8)$ & \\
\hline Voiding difficulty & $13(12.7)$ & $10(11.5)$ & \\
\hline Purulent or turbid discharge $\mathrm{a}^{\mathrm{a}}$ & $9(8.8)$ & $9(10.3)$ & \\
\hline Wound infection & $6(5.9)$ & $1(1.1)$ & \\
\hline Chylous ascites & $3(2.9)$ & $3(3.4)$ & \\
\hline Arrhythmia & $3(2.9)$ & $1(1.1)$ & \\
\hline EPSBO & $2(2.0)$ & $7(8.0)$ & \\
\hline Pneumonia & $0(0)$ & $1(1.1)$ & \\
\hline Grade IIla & $1(1.0)$ & $2(2.3)$ & \\
\hline $\begin{array}{l}\text { Pelvic abscess associated with } \\
\text { anastomotic leakage }\end{array}$ & $1(1.0)$ & $2(2.3)$ & \\
\hline Grade IIIb & $2(2.0)$ & $1(1.1)$ & \\
\hline Anastomotic leakage & $1(1.0)$ & $0(0)$ & \\
\hline Bile peritonitis ${ }^{b}$ & $1(1.0)$ & $0(0)$ & \\
\hline Ileostomy stricture & $0(0)$ & $1(1.1)$ & \\
\hline Complication $\geq$ grade III & $3(2.9)$ & $3(3.4)$ & 0.827 \\
\hline Overall complication & 39 (38.2) & $35(40.2)$ & 0.780 \\
\hline Readmission & $3(2.9)$ & $0(0)$ & 0.155 \\
\hline
\end{tabular}

Values are presented as number (\%) unless otherwise denoted.

EPSBO, early postoperative small bowel obstruction.

${ }^{a}$ Considered as purulent or turbid discharge through the intra-abdominal drainage tube. ${ }^{b}$ Due to gallbladder perforation. 


\section{Coloproctology Do Hoe Ku, et al}

Table 5. Wound complications in the specimen extraction site

\begin{tabular}{lccc}
\hline Variable & $\begin{array}{c}\text { Midline } \\
\text { group } \\
(\mathrm{n}=102)\end{array}$ & $\begin{array}{c}\text { Transverse } \\
\text { group } \\
(\mathrm{n}=87)\end{array}$ & P-value \\
\hline Overall incisional hernia & $22(21.6)$ & $23(26.4)$ & 0.494 \\
\hline Incisional hernia with TS & $18 / 46(39.1)$ & $22 / 62(35.5)$ & 0.840 \\
\hline Incisional hernia without TS & $4 / 56(7.1)$ & $1 / 25(4.0)$ & 0.507 \\
Overall wound infection & $26(25.5)$ & $15(17.2)$ & 0.232 \\
Grade $\mathrm{I}^{\mathrm{b}}$ & $22(21.6)$ & $13(14.9)$ & 0.228 \\
Grade $\|^{\mathrm{b}}$ & $4(3.9)$ & $2(2.3)$ & 0.689 \\
Wound infection with TS $^{\mathrm{c}}$ & $20 / 46(43.5)$ & $14 / 62(22.6)$ & 0.035 \\
Grade $\mathrm{I}^{\mathrm{b}}$ & $18 / 46(39.1)$ & $12 / 62(19.4)$ & 0.023 \\
Grade $\|^{\mathrm{b}}$ & $2 / 46(4.4)$ & $2 / 62(3.2)$ & 0.643 \\
Wound infection without TS $^{\mathrm{b}}$ & $6 / 56(10.7)$ & $1 / 25(4.0)$ & 0.300 \\
Grade $\mathrm{I}^{\mathrm{b}}$ & $4 / 56(7.1)$ & $1 / 25(4.0)$ & 0.508 \\
Grade $\|^{\mathrm{b}}$ & $2 / 56(3.6)$ & $0 / 25(0.0)$ & 0.475 \\
\hline
\end{tabular}

Values are presented as number (\%) unless otherwise denoted.

TS, temporary stoma.

aComparison of specimen extraction site hernia between patients with temporary stoma and those without, $\mathrm{P}<0.001 .{ }^{\mathrm{b}} \mathrm{Grade}$ I infection was defined as wound infection treated by opening the wound; Grade II infection was defined as wound infection treated by opening of the wound and antibiotics due to combined phlegmonous infection. 'Comparison of wound infection in specimen extraction site between patients with temporary stoma and those without, $\mathrm{P}<0.001$.

midline group $(\mathrm{n}=46)$ was comparable to that in the transverse group $(n=62)$ in the patients who underwent SESS $(P=0.643)$ (Table 5).

\section{DISCUSSION}

While a low transverse incision was reported to be the preferred extraction site during LRCS [17-19], the use of a low midline incision as an SES has rarely been reported in LRCS. In this study, the incisional hernia rate in the SES following LRCS was not significantly different between the low midline and low transverse incisions. In addition, among the 81 patients who did not undergo SESS, incisional hernia rates of $7.1 \%$ and $4.0 \%$ at the SES in the low midline incision and low transverse incision groups, respectively, were comparable. There was no significant increase in the wound infection rate in the low midline incision group following LRCS; however, in the 108 patients who underwent SESS, after stoma closure, the wound infection rate with a low midline incision was approximately $44 \%$ and significantly higher than that with the low transverse incision of approximately $23 \%$.

A temporary diverting stoma was performed in $35 \%$ to $80 \%$ of patients who underwent LRCS $[11,24]$, and the possibility of additional abdominal incision for temporary stoma is high in addition to the incision for specimen extraction. Previous studies [17-21] have reported that the SESS is performed through the right (left) low abdomen or umbilical site to maintain the advantages of minimally invasive surgery by reducing the need for an additional minilaparotomy for temporary stoma during LRCS. The present study results also showed that 108 of 114 patients (94.7\%) who required temporary stoma had stoma formation through an SES, and the incidence of the requirement for an additional mini-laparotomy for temporary stoma was comparable between the 2 groups. This finding suggested that a low midline incision in LRCS may also have the advantage of reducing the requirement for an additional mini-laparotomy for temporary stoma, same as that for a low transverse incision. In addition, consistent with the study of Wang et al. [17], who reported no increase in the prevalence of peristomal morbidity in patients who underwent SESS, no serious complications of peristomal wound occurred in our midline group.

We defined incisional hernia as an abdominal wall gap regardless of clinical symptoms on computed tomography. Based on computed tomographic findings, the incidence of overall incisional hernia of the SES in the present study was $21.6 \%$ in the midline group and $26.4 \%$ in the transverse group. Although the incisional hernia rate in the SES in our study was higher than that reported in a previous study [25] of approximately $10 \%$ in low midline or low transverse incision, it is presumed that in $50 \%$ or more of patients the specimen was extracted via the temporary stoma site, which may be a risk factor of incisional hernia after temporary stoma closure $[25,26]$. However, the comparable incisional hernia rates after LRCS between the 2 groups in our study were somewhat different from those reported in a systematic review [10]. The systematic review reported that the incisional hernia rate in the midline incision was 3 times higher than that in transverse incision. Because abdominal wound closure was mostly performed by the attending surgeon in our institution, the similar incisional hernia rates in both groups in our study may be explained by the study of Navaratnam et al. [27], who reported the incidence of incisional hernia is minimized even in the midline incision using the standardized technique. However, some studies $[9,28]$ denoted that midline incision has a higher incisional hernia rate even when using the standardized technique for wound closure during laparoscopic colectomy.

Benlice et al. [25] reported that the incisional hernia rate was significantly increased by 28 times in SESS. In concordance with this study, our results demonstrated an incisional hernia rate of $37 \%$ in patients where mini-laparotomy is used for synchronous temporary stoma formation compared with that of $6 \%$ in the patients where mini-laparotomy is only used for specimen extraction $(\mathrm{P}<0.001)$. However, when the SES is not used for temporary stoma, our incisional hernia rates of the SES were $7.1 \%$ and $4.0 \%$ in the low midline and low transverse incisions, respectively $(\mathrm{P}=$ 0.507), and these results are similar to those of previous studies reporting an incisional hernia rate of approximately $10 \%$ in low midline incision and approximately $4 \%$ in low transverse incision following LRCS $[9,25,28]$. Based on these results, with respect to incisional hernia, low midline incision, if not used for temporary 
stoma formation, may be used as the SES in low transverse incision during LRCS.

In this study, the overall wound infection rate in the SES was not different statistically in $25.5 \%$ of the midline incision group and $17.2 \%$ of the transverse group $(\mathrm{P}=0.232)$. However, in our 108 patients who underwent SESS, the wound infection rate after stoma closure in the SES was significantly higher in the midline group (43.5\%) than in the transverse group (22.6\%) $(\mathrm{P}=0.035)$. Previous reports $[8,29]$ mention that the midline incision has the potential for impaired blood supply to the wound site during wound healing. In this study, it was postulated that wound infection was a higher rate in low midline incision than in low transverse incision in patients with SESS by the hypothesis that impaired blood supply to the low midline wound site combined with wound contamination during stoma closure may increase the likelihood of wound infection. In contrast to a recent meta-analysis [30], in which the wound infection rate was less than $10 \%$ following stoma closure in patients with rectal cancer, the wound infection rate of $31.5 \%$ after stoma closure in our 108 patients who underwent SESS seemed to be quite high. However, the wound infection rate after stoma closure in patients who underwent SESS in this study was interpreted on considering that the wound infection rate after stoma closure can reach $36 \%$ and may be higher than the usual rate reported in previous literature [31]. Another factor to consider about this high wound infection rate is an increased susceptibility to the formation of dead space due to myofascial dissection during wound closure, which may lead to an increased accumulation of exudate. Therefore, we attempted to minimize the accumulation of wound fluid and dead space formation by wound drainage and 2-layer subcutaneous suture during stoma closure in cases of SESS.

The authors' surgical technique is somewhat different from the conventional 5-port laparoscopic surgery in that it is a modified 4-port technique in which a mini-laparotomy is first carried out. Although the port location of the transverse group was similar to that of conventional LRCS, the main procedure for dissection and transection of the rectum in the midline group was performed through the glove port located in the low midline abdomen. Our study showed that in a comparison of surgical technique related outcomes, the midline group was significantly superior to the transverse group in the frequency of temporary stoma formation and splenic flexure mobilization. Although disagreement exists as to which port to use for major surgical manipulation in LRCS [32], our findings suggest that the use of a working port located in the low midline abdomen has a positive effect on the surgical outcome.

This study has several limitations. First, although no significant difference was found between the clinical characteristics of the 2 groups, a possibility of bias exists in patient selection due to the retrospective study design. Second, because most specimens were extracted through the midline abdomen or right (left) low abdomen at our institution, this study did not include data on Pfan- nenstiel incisions with advantages in terms of wound complications $[8,33]$. Third, this study did not include data on medical comorbidities, such as diabetes mellitus and obstructive lung disease that could affect wound complications after LRCS due to the limitations of the retrospective study. Despite these limitations, to the best of our knowledge, this is the first study to report a low midline incision as an SES in LRCS.

In conclusion, in relation to incisional hernia and wound infection at the SES, a low midline incision may be used as a low transverse incision in patients without temporary stoma in LRCS. However, considering the high rates of incisional hernia and wound infection after stoma closure in patients who underwent SESS through low midline or low transverse incisions in this study, SESS should be performed with caution in LRCS.

\section{CONFLICT OF INTEREST}

No potential conflict of interest relevant to this article was reported.

\section{REFERENCES}

1. Benlice C, Stocchi L, Sapci I, Gorgun E, Kessler H, Liska D, et al. Impact of the extraction-site location on wound infections after laparoscopic colorectal resection. Am J Surg 2019;217:502-6.

2. Singh R, Omiccioli A, Hegge S, McKinley C. Does the extractionsite location in laparoscopic colorectal surgery have an impact on incisional hernia rates? Surg Endosc 2008;22:2596-600.

3. Samia H, Lawrence J, Nobel T, Stein S, Champagne BJ, Delaney CP. Extraction site location and incisional hernias after laparoscopic colorectal surgery: should we be avoiding the midline? Am J Surg 2013;205:264-7.

4. Steinert R, Lippert H, Reymond MA. Tumor cell dissemination during laparoscopy: prevention and therapeutic opportunities. Dig Surg 2002;19:464-72.

5. Morita Y, Yamaguchi S, Ishii T, Tashiro J, Kondo H, Suzuki A, et al. Does transumbilical incision increase incisional hernia at the extraction site of laparoscopic anterior resection? Am J Surg 2015;209:1048-52.

6. Lim SW, Huh JW, Kim YJ, Kim HR. Vertical transumbilical incision versus left lower transverse incision for specimen retrieval during laparoscopic colorectal surgery. Tech Coloproctol 2013;17: 59-65.

7. Shin JY. Right lower transverse incision versus vertical transumbilical incision for laparoscopic specimen extraction in patients with left-sided colorectal cancer: a comparative study of two mini-laparotomy techniques. World J Surg Oncol 2016;14:274.

8. Orcutt ST, Balentine CJ, Marshall CL, Robinson CN, Anaya DA, Artinyan A, et al. Use of a Pfannenstiel incision in minimally invasive colorectal cancer surgery is associated with a lower risk of wound complications. Tech Coloproctol 2012;16:127-32.

9. Lee L, Mata J, Droeser RA, Kaneva P, Liberman S, Charlebois P, et 
al. Incisional hernia after midline versus transverse specimen extraction incision: a randomized trial in patients undergoing laparoscopic colectomy. Ann Surg 2018;268:41-7.

10. Lee L, Abou-Khalil M, Liberman S, Boutros M, Fried GM, Feldman LS. Incidence of incisional hernia in the specimen extraction site for laparoscopic colorectal surgery: systematic review and meta-analysis. Surg Endosc 2017;31:5083-93.

11. van der Pas MH, Haglind E, Cuesta MA, Fürst A, Lacy AM, Hop WC, et al. Laparoscopic versus open surgery for rectal cancer (COLOR II): short-term outcomes of a randomised, phase 3 trial. Lancet Oncol 2013;14:210-8.

12. Kang SB, Park JW, Jeong SY, Nam BH, Choi HS, Kim DW, et al. Open versus laparoscopic surgery for mid or low rectal cancer after neoadjuvant chemoradiotherapy (COREAN trial): short-term outcomes of an open-label randomised controlled trial. Lancet Oncol 2010;11:637-45.

13. Sun Z, Kim J, Adam MA, Nussbaum DP, Speicher PJ, Mantyh CR, et al. Minimally invasive versus open low anterior resection: equivalent survival in a national analysis of 14,033 patients with rectal cancer. Ann Surg 2016;263:1152-8.

14. Denost Q, Adam JP, Rullier A, Buscail E, Laurent C, Rullier E. Perineal transanal approach: a new standard for laparoscopic sphincter-saving resection in low rectal cancer, a randomized trial. Ann Surg 2014;260:993-9.

15. Velthuis S, Nieuwenhuis DH, Ruijter TE, Cuesta MA, Bonjer HJ, Sietses C. Transanal versus traditional laparoscopic total mesorectal excision for rectal carcinoma. Surg Endosc 2014;28:3494-9.

16. Ng HI, Sun WQ, Zhao XM, Jin L, Shen XX, Zhang ZT, et al. Outcomes of trans-anal natural orifice specimen extraction combined with laparoscopic anterior resection for sigmoid and rectal carcinoma: an observational study. Medicine (Baltimore) 2018;97: e12347.

17. Wang P, Liang JW, Zhou HT, Wang Z, Zhou ZX. Surgical specimen extraction via a prophylactic ileostomy procedure: a minimally invasive technique for laparoscopic rectal cancer surgery. World J Gastroenterol 2018;24:104-11.

18. Karakayali FY, Tezcaner T, Moray G. Specimen extraction from the defunctioning ileostomy site or Pfannenstiel incision during total laparoscopic low anterior resection for rectal cancer. J Laparoendosc Adv Surg Tech A 2015;25:380-5.

19. Yoo SB, Jeong SY, Lim SB, Park JW, Choi HS, Oh JH. Left-sided ileostomy at specimen extraction site in laparoscopic-assisted low anterior resection for rectal cancer. J Laparoendosc Adv Surg Tech A 2013;23:22-5.

20. d'Alessandro A, Kari N, Alameh A, Pasquier N, Tarhini A, Vinson Bonnet B, et al. Preliminary experience with umbilical stoma in transumbilical single-port colorectal surgery. Tech Coloproctol 2018;22:301-4.
21. Miyo M, Takemasa I, Hata T, Mizushima T, Doki Y, Mori M. Safety and feasibility of umbilical diverting loop ileostomy for patients with rectal tumor. World J Surg 2017;41:3205-11.

22. Mimica Z, Pogorelić Z, Perko Z, Srsen D, Stipić R, Dujmović D. Effect of surgical incision on pain and respiratory function after abdominal surgery: a randomized clinical trial. Hepatogastroenterology 2007;54:2216-20.

23. Dindo D, Demartines N, Clavien PA. Classification of surgical complications: a new proposal with evaluation in a cohort of 6336 patients and results of a survey. Ann Surg 2004;240:205-13.

24. Stevenson AR, Solomon MJ, Lumley JW, Hewett P, Clouston AD, Gebski VJ, et al. Effect of laparoscopic-assisted resection vs open resection on pathological outcomes in rectal cancer: the ALaCaRT randomized clinical trial. JAMA 2015;314:1356-63.

25. Benlice C, Stocchi L, Costedio MM, Gorgun E, Kessler H. Impact of the specific extraction-site location on the risk of incisional hernia after laparoscopic colorectal resection. Dis Colon Rectum 2016;59:743-50.

26. Bhangu A, Nepogodiev D, Futaba K; West Midlands Research Collaborative. Systematic review and meta-analysis of the incidence of incisional hernia at the site of stoma closure. World J Surg 2012;36:973-83.

27. Navaratnam AV, Ariyaratnam R, Smart NJ, Parker M, Motson RW, Arulampalam TH. Incisional hernia rate after laparoscopic colorectal resection is reduced with standardisation of specimen extraction. Ann R Coll Surg Engl 2015;97:17-21.

28. Winslow ER, Fleshman JW, Birnbaum EH, Brunt LM. Wound complications of laparoscopic vs open colectomy. Surg Endosc 2002;16:1420-5.

29. Burger JW, van't Riet M, Jeekel J. Abdominal incisions: techniques and postoperative complications. Scand J Surg 2002;91:315-21.

30. Hajibandeh S, Hajibandeh S, Sarma DR, East J, Zaman S, Mankotia R, et al. Meta-analysis of temporary loop ileostomy closure during or after adjuvant chemotherapy following rectal cancer resection: the dilemma remains. Int J Colorectal Dis 2019;34:11519.

31. Liang MK, Li LT, Avellaneda A, Moffett JM, Hicks SC, Awad SS. Outcomes and predictors of incisional surgical site infection in stoma reversal. JAMA Surg 2013;148:183-9.

32. Miskovic D, Foster J, Agha A, Delaney CP, Francis N, Hasegawa H, et al. Standardization of laparoscopic total mesorectal excision for rectal cancer: a structured international expert consensus. Ann Surg 2015;261:716-22.

33. DeSouza A, Domajnko B, Park J, Marecik S, Prasad L, Abcarian H. Incisional hernia, midline versus low transverse incision: what is the ideal incision for specimen extraction and hand-assisted laparoscopy? Surg Endosc 2011;25:1031-6. 